\title{
The Genesis of Representation
}

\author{
W A Cameron
}

Glasgow, UK

\begin{abstract}
In language, humans describe their world and can construct a narrative of their own becoming. But the concepts within which the emergence of linguistic man may be described are, themselves, contingent on thee accidents of that emergence Thee human world is objective but the terms of that objectivity are contingent on human morphology and the accidents of its evolution.
\end{abstract}

Keywords: language; evolution; emergence; concepts

Acknowledgement: Text

\section{Introduction}

The content of linguistic representations, shared within human concepts of a human world, has naturally and contingently evolved. Content, what it is that an environment presents to an organism and causes it to act, emerged with life itself. Such content can only be described in the concepts of an observer within an observer's theory of cognition for an organism in its environment. For primitive organisms, in which action is reflexive, proximal sensory input may be causally associated with distal affordance, and what is taken as represented may be chosen by a theorist. For those organisms, whether octopus, bird, dolphin, ape or human, in which representations are realised in informational structures which combine modes of perception to model space and time, what is represented may be some substance that has affordance for the organism, and neural science is now giving us some insight into how this may be realised. The world described by an observer is the human objective world framed in human concepts, some of which, like danger, have been inherited from our hominoid ancestors and some of which, like representation or money, have culturally emerged. For each organism its cognitive structure models its world in terms of its concepts, and this world is real for it. However, in general this world cannot be described, though it may be inferred.

\section{Explanation}

By their living, humans have within language constructed an objective world of substances, actions and events which are shared in the terms of linguistic concepts, and the items of this objective world include organisms of a vast range of cognitive complexity. One of mankind's linguistic practices is the construction of explanations for the behaviour of organisms including man. One class of such explanations is based on 
a theory of agency which includes input to the organism from an environment, and cognitive mechanisms within the organism by which such input causes action. It is a platitude of evolution that those species which have survived are those with cognitive mechanisms which, ceteris paribus, cause resultant action that is appropriate for the survival of the organism or its reproduction. Another is based on a theory of mind, citing beliefs and desires, but this is not within the concern of this essay

\section{Perception}

An observer, writing within her conceptual scheme for an audience who share that scheme, may describe the behaviour of any organism as physically caused by events in its environment. Changes in contiguous states of the environment cause changes in states of the organism and changes in these states cause movements of the organism which may be interpreted as action about such changes. For any given species, particular contiguous properties will be relevant and examples of these include: a chemical gradient across the organism, orientation of incident electromagnetic radiation, tactile pressure and gravitational orientation. Static or dynamic combinations of such contiguity may be relevant but the organism can only be affected by change in contiguous properties. Distal properties may be seen by the observer as causing contiguous phenomena in an observer's model of the environment of the organism, but an organism is a closed substance and can only be affected by energetic impingements on its surfaces. Gibson (1968) describes the variety of physical processes which may be involved in such energetic transduction.

\section{Natural Representation}

An observer may have the technology to pick out some component or components of an organism with properties that are varied by energetic impingements from the environment and which by combinatorial processing cause movements of the organism which are appropriate for the its survival or reproduction. Such components have an cognitive function for the organism and, in the terminology of Dretske (1988: 62), their function may be seen as naturally representational since the state of the component, which may be in any physical modality used by the interconnecting cognitive mechanisms, stands in either for the form of energetic impingement which is relevant for this species or the affordance which it signifies. For many organisms this is as far as a theorist needs to go. That reproduction needs a mate, that food flies past and that predators make a noise are all facts known to the theorist, but do not change the structure of the model outlined above. 'Representation' here is only used realistically in connection with specific form of energetic impingements that are relevant to the cognitive mechanisms of a given species. Any interpretation in terms of distal phenomena must be instrumental since it will be derived from an ecological theory of the world for the organism. For organisms of this level the notion of recognition does not arise. Many organisms do learn, in that some current representational states depend on past incident phenomena, but all that this learning does is to extend the range of relevant incident phenomena without any need to invoke a cognitive structure of an environment distal to the organism.

\section{Concepts}

In all organisms, the mechanisms by which incident phenomena change representational states can be thought of as perception, but for those organisms in which one class of behaviour is associated with one sort of incident phenomena the term does little work. However, in some organisms more than one mode of stimulation is involved in a class of behaviour. Attention may be reflexively directed by some impingement towards some spatial location but if, in virtue of previous experience, neural structures exist within the organism which are tuned to particular multi-modal combinations of sensory impingement and action the organism may be said to recognise some aspect of the world which is associated with such combinations. Eyes, ears, hands or claws, and wings may all be associated in such combinations; what is recognised is a gestalt of stimulation and action which, in an observer's theory, may be associated with affordance and associated action. 
Description and explanation of behaviour as complex as this requires mention of a world distal to the organism, but this is a requirement on the theorist. What is explanatorily relevant is whether cognitive structures exist within the creature which are tuned to the combinatorial dynamics of optical, tactile and proprioceptive impingements on the organism, as the chemistry at the base of the flagella of a bacterium is tuned to the chemical gradient of its environment. Since these cognitive structures are objective phenomena they are in principle as available for observation as representational properties within a primitive organism and recent work in neural science has demonstrated their existence in man and monkey. See for example, Gallese and Lakoff (2005) for recent work in this area.

This is a big leap. The simple representational states that support the reflexive actions of a bacterium, the phototropism of a flower, the snapping of a Venus fly trap or the snatching of a frog all rely for their function of representation on a causal link between incident phenomena and implicit representation of that phenomena. Correlation of such phenomena with nourishment or reproduction due to distal effects is built in by evolution. Attribution of representation to recognitional states only makes explanatory sense when recognition is seen as of complex distal phenomena and these phenomena can only be described in the conceptual structure of an observer. For the organism all that has changed is that complex temporal correlations between incident phenomena cause complex patterns of behaviour. This applies to any form of cognition in which the co-ordination of sensory modalities reacts to phenomena in space and time. Such co-ordination is observed in creatures as diverse as cephalopods, cetaceans, birds and primates and it may be conjectured that in all such creatures this co-ordination leads to the cognitive modeling of a spatiotemporal world. I doubt if there is a clear boundary, Tirassa, Carassa et al. (2000) suggest that this is the class of animals which represent their world and that further ecological research is needed.

\section{The Emergence of Language}

Mather and Anderson (1998) describe octopus behaviour which seems clearly to involve a world of space and time but with sensory modalities very different from human's, it is likely that little of that world overlaps with the human objective world. We can speculate that somehow they represent items of, and actions in, their world but what these are may not be describable in human linguistic concepts. However, chimpanzees are very like us in their sensory modalities and we share a recent common ancestry. One can therefore speculate that those hominoid concepts which were referred to in the early speech of our hominid ancestors were within the conceptual structure of the objective world in terms of which we now interpret the behaviour of an ape, a dolphin or an octopus. From this early speech current human language has emerged. It is within that language that we have developed an objective world of things, and an objective world of science in which we can describe the properties of the environment of a bacterium, and those features of the sea bed which might be visible or tactile to an octopus.

\section{$7 \quad$ Linguistic Concepts}

It is in the concepts of the human objective world that we describe states of an organism as representing features of a world, but each human observer is herself an organism and the concepts to which she refers in such description are from the human construction of a human world. Each person is embodied in a an organic agency which is informationally closed apart from the proximal impingements of the human senses. However, humans are different in that the spatiotemporal world constructed by complex sensory modalities is shared in language so that conceptual structures exist beyond their application in action. Humans have invented the notion of conventional representation [Dretske (op cit.:52)] within which pictures represent people, maps represent terrain and sentences represent propositions, or states of affairs.

From this use of 'representation' and the objective world of empirical science our use of 'representation' to describe a critical function in organic agency has developed, and it is from this latter sort of representation that our complex cognitive structures and concepts of space and time have emerged. A world of things for any organism must be a construct from the sensory modalities of that organism as the 
autonomy of any organism is defined by its bounding surface. Due to this, the acquisition of information by any organism is restrained to the proximality of its senses, and its recognitions are of items of that world which can not be described but only shown. Such recognitions may be realised in specific neural structures and action will result from combinatorial processing of such structures. Such structures may be interpreted as modelling a world for the creature.

\section{The Hermeneutic Circle}

We have come full circle. As far as we humans can tell, mankind is the only species with language and through language we have developed an objective view of our environment on planet Earth. Through the objective science of biology we have come to appreciate that we are but one species in a rich diversity of organic life, and may speculate that this diversity has contingently evolved from an accidental beginning. Fossil evidence objectively analysed within palaeontology supports this hypothesis and we must conclude that the objectivity within which we may learn to appreciate the contingency of our becoming is itself a contingent outcome. Had primates not evolved, the dryopithecine strand that led to human kind would not have evolved. Not only would this story not have been true, but the concepts in which this story may be told would not be available. The objective structures within which we can describe our contingent becoming are themselves contingent on that becoming.

\section{References}

Dretske, F. I. (1988). Explaining Behavior: Reasons in a World of Causes. Cambridge, MIT a Bradford Book.

Gallese, V. and G. Lakoff (2005). "The brains concepts: The role of the sensory motor system in conceptual knowledge." Cognitive Neuropsychology prEview.

Gibson, J. J. (1968). The Senses Considered as Perceptual Systems. London, George Allen \& Unwin.

Mather, J. A. and R. C. Anderson (1998). What behavior can we expect of octopuses? The Cephalopod Page. J. B. Wood. http://www.dal.ca/ ceph/TCP/behavior.html.

Tirassa, M., A. Carassa, et al. (2000). A theoretical framework for the study of spatial cognition. Spatial Cognition. Foundations and Appplications. S. O'Nuallain. Amsterdam/Philadelphia, Benjamins: 19-31.

I 